\title{
Isolated Fe sites in Metal Organic Framework catalyze the direct conversion of methane to methanol
}

Dmitrii Osadchii, Alma Itzel Olivos Suarez, Ágnes Szécsényi, Guanna Li, Maxim A. Nasalevich, A lulian Dugulan, Pablo Serra-Crespo, Emiel J. M. Hensen, Sergey L. Veber, Matvey V. Fedin, Gopinathan Sankar, Evgeny A Pidko, and Jorge Gascon

ACS Catal., Just Accepted Manuscript • DOI: 10.1021/acscatal.8b00505 • Publication Date (Web): 10 May 2018

Downloaded from http://pubs.acs.org on May 15, 2018

\section{Just Accepted}

"Just Accepted" manuscripts have been peer-reviewed and accepted for publication. They are posted online prior to technical editing, formatting for publication and author proofing. The American Chemical Society provides "Just Accepted" as a service to the research community to expedite the dissemination of scientific material as soon as possible after acceptance. "Just Accepted" manuscripts appear in full in PDF format accompanied by an HTML abstract. "Just Accepted" manuscripts have been fully peer reviewed, but should not be considered the official version of record. They are citable by the Digital Object Identifier (DOI®). "Just Accepted" is an optional service offered to authors. Therefore, the "Just Accepted" Web site may not include all articles that will be published in the journal. After a manuscript is technically edited and formatted, it will be removed from the "Just Accepted" Web site and published as an ASAP article. Note that technical editing may introduce minor changes to the manuscript text and/or graphics which could affect content, and all legal disclaimers and ethical guidelines that apply to the journal pertain. ACS cannot be held responsible for errors or consequences arising from the use of information contained in these "Just Accepted" manuscripts. 


\section{INTRODUCTION}

Targeting the exquisite reactivity and selectivity that methane monooxygenase enzymes display for the conversion of methane to methanol requires the design of a chemical model able to combine characteristics both from the organic and inorganic materials fields. New organicinorganic synthetic solids, such as metal organic frameworks (MOFs), offer a much higher degree of synthetic control and may become ideal solids for the synthesis of model methane activation catalysts (Fig. 1). ${ }^{1}$ The current design of active sites in MOF-based catalysts is dominated by homogeneous catalysis and coordination chemistry strategies where the classical limitations of the respective approaches are not yet overcome.
Researchers have tried to emulate similar enzyme-like active sites within well-defined molecular ligand architectures and synthetic solids to address the challenge ${ }^{2}$ to selectively activate methane. ${ }^{3}$ In nature, the methane monooxygenase enzymes (MMOs) are able to directly and selectively oxidise methane to methanol (Fig. 1a) under mild aqueous conditions. ${ }^{4}$ In the soluble version of the MMOs, the active site is a dimeric Fe species in the hydroxylase subunit (Fig. 1b). Two additional components mediate an electron transfer chain to reduce the Fe(III)$\mathrm{Fe}(\mathrm{III})$ antiferromagnetically coupled, high spin, dimeric site to a Fe(II)-Fe(II) active species that effectively activates oxygen to produce a high valent metal-oxo intermediate. This highly oxidative intermediate is responsible for the activation of the $\mathrm{C}-\mathrm{H}$ bond in methane via hydrogen abstraction. ${ }^{5}$ Worth noticing is that the sMMO relies on the redox accessibility of the Fe dimer: the reduction step 
toward $\mathrm{Fe}(\mathrm{II})-\mathrm{Fe}(\mathrm{II})$ species and the high valent $\mathrm{Fe}(\mathrm{IV})$ $\mathrm{Fe}(\mathrm{IV})$. It has been proven that the hydroxylase component retains catalytic activity when the 2-electron donor chain is substituted by $\mathrm{H}_{2} \mathrm{O}_{2}$, thus proving the independence of these two events. ${ }^{6}$ Notably, the high selectivity of this system is ensured by the encapsulation of methane in the nonpolar environment of the reaction centre that promotes the rebound mechanism and by the noncovalent interactions with the protein matrix that help product removal to prevent side-reactions. ${ }^{5 \mathrm{~b}}$ In spite of these great efforts, catalysts designed on basis of the enzyme still need to overcome two major milestones, namely to avoid dissociation of the iron dimeric species after the catalytic cycle and to fully reproduce the magnetic properties of the $\mathrm{Fe}(\mathrm{III})-\mathrm{Fe}(\mathrm{III})$ dimer, postulated to be crucial on reactivity. ${ }^{7}$ Since the pioneering work of Panov, ${ }^{8}$ zeolites have received a great deal of attention. ${ }^{9}$ However, the intrinsic hydrophilic nature and the relatively poor degree of tuneability in most pure inorganic matrices, including zeolites, lead to ill-defined active sites and to strong adsorption of methanol inside the zeolite pores, thus effectively creating stoichiometric reactants rather than catalysts. As demonstrated in this report, these drawbacks can be circumvented by making use of hybrid materials. Here, we report that the controlled incorporation of isolated Fe units into an Al-based MOF results in methane oxidation catalysts that display a high activity and selectivity.

\section{ISOLATING CATALYTIC ACTIVE SITES}

Targeting the isolation of $\mathrm{Fe}_{\text {oxo }}$ sites in an environment resembling that of the enzymatic systems within a MOF structure, we selected the MIL-53(Al) structure as the support matrix because it is built from well-defined chains of non-redox active $\mathrm{AlO}_{6}$ octahedra connected via trans-bridging $\mathrm{OH}$ ions and carboxylate moieties from the bridging 1,4-benzene-dicarboxylate linkers (Fig. 1c). ${ }^{10} \mathrm{We}$ speculated that such a metal-oxo chain structure should be able to accommodate isolated Fe species with a coordination environment close to that in the sMMO active site." Furthermore, unlike other bulk porous materials commonly used as catalyst supports, the micropores of MIL-53 are constructed from hydrophobic organic linkers that should favour the rapid desorption of methanol from the reactive centre in an aqueous environment.

We followed two different synthetic pathways for the incorporation of $\mathrm{Fe}$ atoms into the MIL-53(Al) framework: 1) post-synthetic cation exchange using hydrothermally synthesised MIL-53 $(\mathrm{Al})$ and $\mathrm{FeCl}_{3}$ solutions (samples denoted as HTS); and 2) the in situ Fe incorporation via the addition of $\mathrm{FeCl}_{3}$ aliquots during electrochemical synthesis of MIL-53(Al) from an Al-electrode and a terephthalic acid solution (samples denoted as ECS). ${ }^{12}$ The efficiency of the post-synthetic cation exchange procedure was found to depend strongly on the solvent and temperature used. Successful incorporation of $\mathrm{Fe}$ into MIL-53(Al) with preservation of the MOF structure could be achieved only when the cation exchange was carried out in DMF at low temperature $\left(80{ }^{\circ} \mathrm{C}\right)$. In this case, total $\mathrm{Fe}$ incorporation values ranging from 0.15 to 2 weight percent (wt \%) could be achieved. Post-synthetic modification at higher temperature $\left(120{ }^{\circ} \mathrm{C}\right)$ allowed incorporating amounts of $\mathrm{Fe}$ as high as $16.6 \mathrm{wt} \%$, however, at the expense of extraframework $\alpha-\mathrm{Fe}_{2} \mathrm{O}_{3}$ (Fig. S2-S3). Electrochemical synthesis, on the other hand, offered a greater control over Fe incorporation in the range from 0.3 to $5.5 \mathrm{wt} \%$ by only varying the concentration of Fe precursor (see SI Methods for details). Following this method, we were able to achieve a highly homogeneous distribution of Fe sites within the lattice of MIL-53, as evidenced by the results of extensive physicochemical characterisation (vide infra, Fig. 1d-f and Fig. S2-S5). Broad XRD peaks of ECS samples indicate the formation of very small MOF nanoparticles that cluster with each other ${ }^{12-13}$. The agglomeration of these small crystals also eliminates the breathing in the material ${ }^{14}$, so that the ECS materials reside in the large pore form in a wide range of temperatures and pressures.

\section{METHANE REACTIVITY}

The hybrid HTS and ECS materials were tested for catalytic methane oxidation in water using $\mathrm{H}_{2} \mathrm{O}_{2}$ as oxidant under mild reaction conditions, in a procedure similar to that previously employed for pure inorganic Fe-zeolite catalysts. ${ }^{9 \mathrm{~d}}$ While at higher temperatures $\left(>8 \mathrm{o}{ }^{\circ} \mathrm{C}\right)$ the MIL-53 $(\mathrm{Al}, \mathrm{Fe})$ catalysts were not stable in aqueous $\mathrm{H}_{2} \mathrm{O}_{2}$, reactions performed at temperatures below $60{ }^{\circ} \mathrm{C}$ showed the selective formation of methanol along with traces of over-oxidation products. Analysis of the liquids (by ${ }^{1} \mathrm{H}$ NMR) and of the gaseous head space of the reaction (GC) did not show other products than methanol, methyl peroxide, formic acid and $\mathrm{CO}_{2}$. Products arising from $\mathrm{C}-\mathrm{C}$ coupling were not observed. In all tests, the optimal selectivity could be obtained at short reaction times (approx. 1 hour) and after 2 hours of reaction the activity decreases seemingly as consequence of the prevailed metal mediated $\mathrm{H}_{2} \mathrm{O}_{2}$ decomposition. Under the optimised conditions, reactions performed with the same catalysts but in lower amounts (Fig. S6) result in higher TONs, that can indicate high diffusional limitations within the MOF catalysts.

The stability of the HTS catalysts with high Fe content (HTS-2 and HTS-3, 5.4 and 16.6 Fe wt \% respectively) turned out to be an issue. Fe leaching into the solution was observed, along with the formation of a higher amount of side products and the presence of dissolved linker in solution, arising from the partial digestion of the framework. Fe incorporation through post synthetic ion exchange may result in the formation of large Fe clusters either as integral part of the framework or as extraframework species (even when those could not be observed by XRD). Indeed, analysis of all Fe containing samples by Raman and Fe K-edge extended X-ray absorption spectroscopies (EXAFS) revealed the presence of $\alpha$ $\mathrm{Fe}_{2} \mathrm{O}_{3}$ in highly loaded samples obtained via postsynthetic ion exchange (Fig. S3, S11-S12, Table S2).

To our delight, the formation of such extra-framework species is suppressed for every sample synthesised through the electrochemical route, even when reaching Fe loadings as high as $5.4 \mathrm{wt} \%$. Characterization of sam- 
A $\mathrm{CH}_{4} \underset{[\mathrm{Fe}] @ \mathrm{CAT}}{\stackrel{\text { oxidant }}{\longrightarrow}} \mathrm{CH}_{3} \mathrm{OH}$
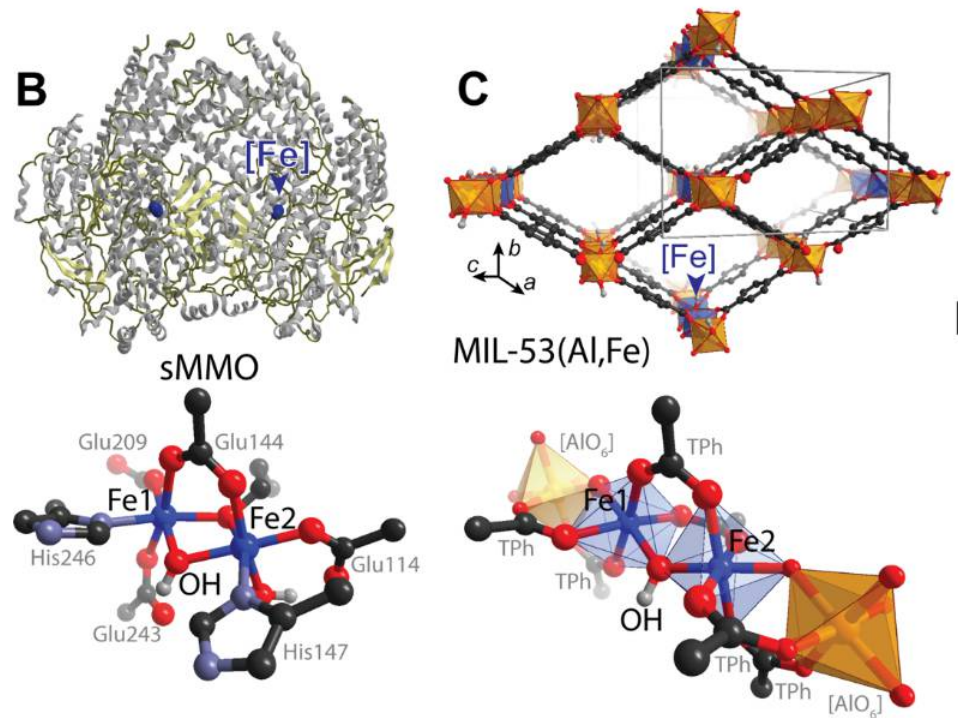
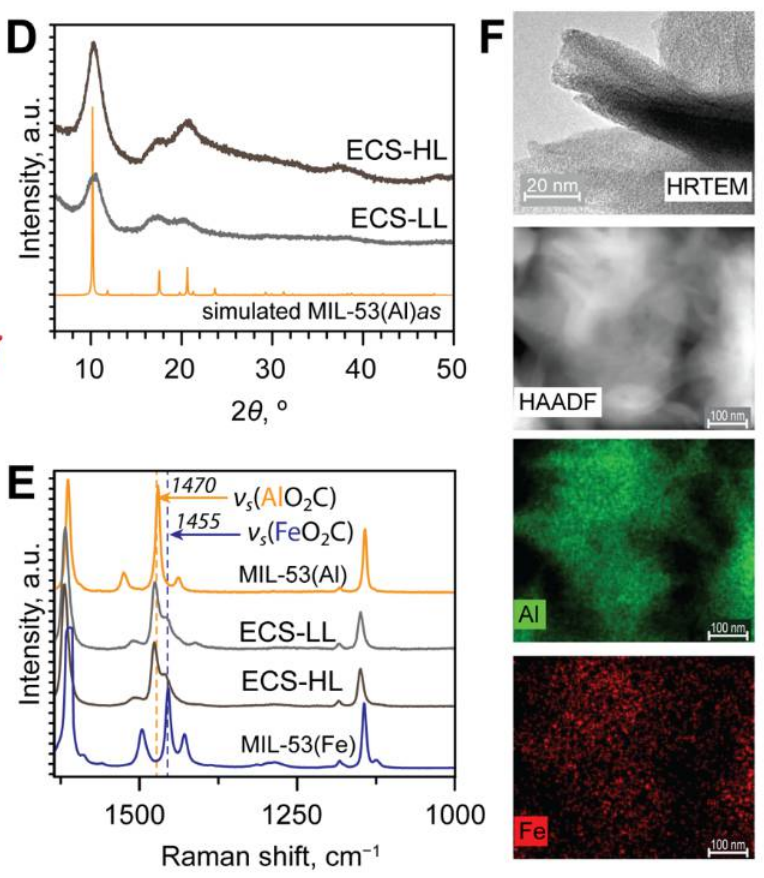

Figure 1. MOF mediated direct methane oxidation. (a) Reaction scheme for the direct catalytic methane oxidation. (b) Structure of sMMO hydroxylase (MMOH, PDB accession code IMTY) enzyme, where each $\alpha_{2} \beta_{2} \gamma_{2}$ dimer contains two diiron active sites (blue spheres and ball and stick representation). (c) The MIL-53 $(\mathrm{Al}, \mathrm{Fe})$ catalyst with the ball and stick representation of a site-isolated Fe within the MIL-53 octahedral [AlO 6 ] chain. (d) X-ray diffraction (XRD) analysis of the electrochemically synthesized (ECS) samples compared to the simulated XRD patterns of the (as synthesized) MIL-53(Al) structure. (e) Raman spectra of ECS samples compared to the pure MIL-53(Al) and MIL-53 $(\mathrm{Fe})$ phase. Laser wavelength, $785 \mathrm{~nm}$; integration time $60 \mathrm{~s}$. (f) Representative HRTEM and HAADF-STEM of ECS MIL-53(Al,Fe) catalysts with low Fe loading (-LL). The abbreviation -HL is used to denote materials with a high Fe loading $(>5 \mathrm{wt} \%)$.

ples after catalytic testing by means of Raman (Fig. S3e), XRD (Fig. S4) and $\mathrm{CO}_{2}$ adsorption measurements (Table $\mathrm{S} 1$, Fig. $\mathrm{S} 1$ ) shows that electrochemically-synthesised catalysts are stable in the reaction conditions, and iron species reside in the MOF structure without formation of $\mathrm{Fe}_{2} \mathrm{O}_{3}$. Compared to a reference $\mathrm{Fe}^{3+}$ solution, ECS-HL catalyst shows at least 4 times higher activity at the same concentration of iron in the reaction mixture (Fig. S7). In general, the ECS samples display a higher activity than those obtained through post-synthetic ion exchange, with TOFs in the order of $90 \mathrm{~h}^{-1}$ and selectivities to oxygenates of ca. $80 \%$ (Fig. 2a). A comparison between both ECS and HTS samples in terms of particle morphology (TEM) and Fe distribution (HAADF-STEM and energy dispersive Xray spectroscopy (EDX, Fig. S2) reveals a smaller particle size along with a high Fe dispersion for the ECS catalysts. In addition, in the Raman spectra of these samples, the symmetric and asymmetric carboxylate vibrations are the result of the combination of carboxylates attached to either Fe or $\mathrm{Al}$ (Fig. 1e), strengthening the conclusion of a better dispersion of the Fe atoms within the framework.

\section{UNRAVELLING THE CHEMICAL NATURE OF THE CATALYTIC ACTIVE SITES}

In order to reveal the chemical state and local structure of Fe species in the MOF catalysts they were characterized using XAS technique. Fe K-edge XANES spectra show that iron is present in $3^{+}$oxidation state in all samples (according to the edge position at $\sim 7126 \mathrm{eV}$ ). Low intensity of the pre-edge feature indicates octahedral coordination of Fe within the MOFs (Fig. S9). Further information on the state of $\mathrm{Fe}$ in the catalysts was obtained by fitting the EXAFS spectra of the HTS-1, ECS-LL and ECS-HL samples using models including one, two and three $\mathrm{Fe}$ atoms at the octahedral chain of the MIL-53(Al) crystal structure (Fig. $3 \mathrm{~b}$ and Fig. S13). The obtained fitting parameters suggest that Fe sits in an octahedral coordination in the structure of HTS-1 and both ECS samples (Fig. 3b; Table $\left.\mathrm{S}_{3}\right)$. The first peak in the R-space is best fitted by two shells, two $\mathrm{O}$ atoms at 1.95-1.96 $\AA$ and four $\mathrm{O}$ atoms at slightly longer distance (2.01- $2.03 \AA$ ) corresponding to the first coordination sphere around the iron sites. In this model, we assign the $4 \mathrm{O}$ atoms at the equatorial positions to those arising from the two carboxylate-bridging ligands. The apical oxygen atoms are assigned to the expected shorter bridging $\mathrm{Fe}-\mu-\mathrm{O}-\mathrm{Fe}$-bonds. All measured samples show a feature between $\mathrm{R} \sim 2.9$ to $4.0 \AA$ that can be fitted with a combination of different scattering shells arising from the neighbouring carbons (carboxylates from the terephthalic linker) and two metal centres from the octahedral chain. However, the large uncertainty in Debye-Waller factors for these shells indicate a wide range of Fe-ligand bond lengths and it cannot account for the different species considered in our three models. Thus, 

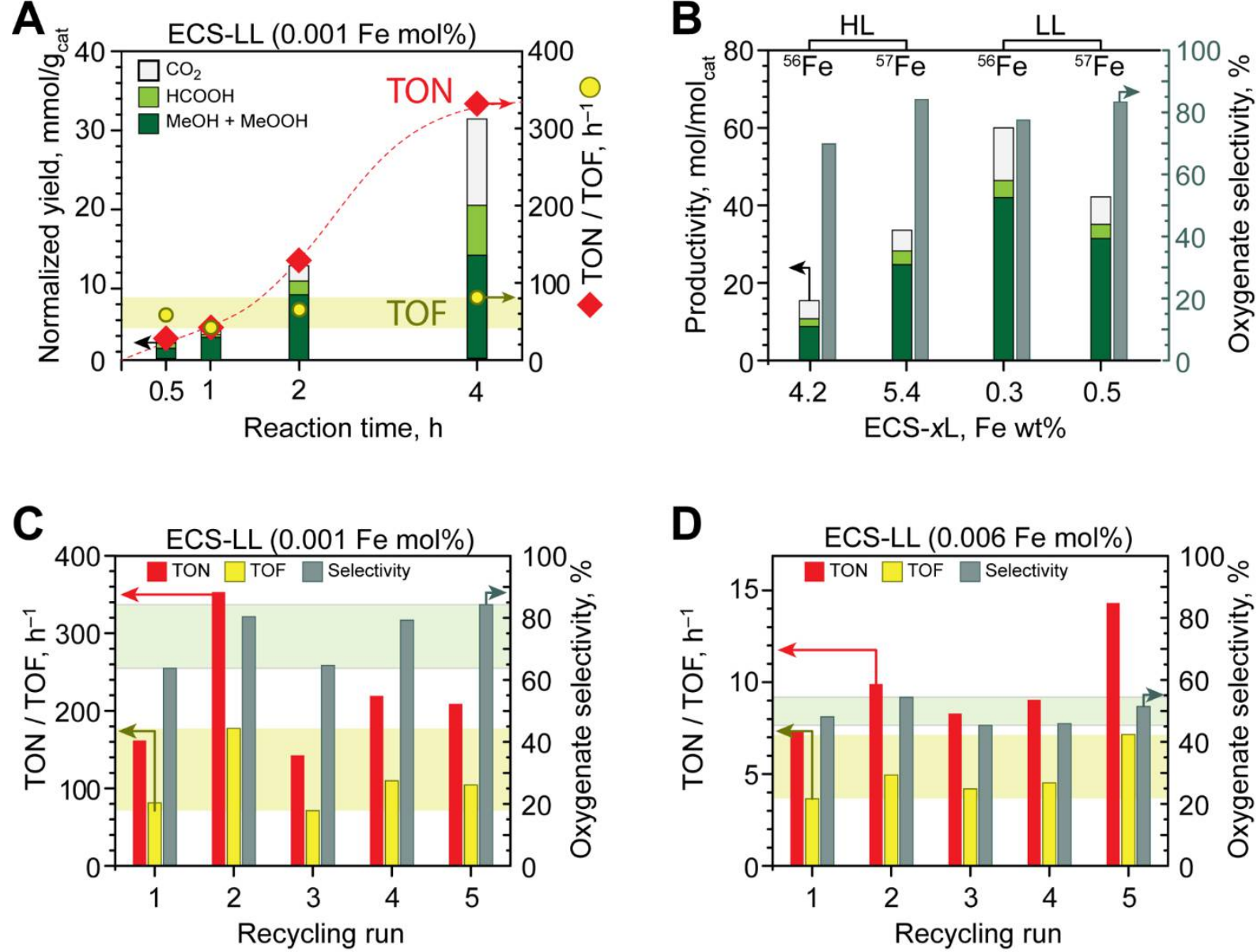

Figure 2. Catalytic oxidation of methane to methanol with $\mathrm{H}_{2} \mathrm{O}_{2}$ by $\mathrm{MIL}-53(\mathrm{Fe}, \mathrm{Al})$. (a) Time-evolution of oxidation products and the respective values of TOF and TON during methane to methanol oxidation in the presence of low concentrations of ECS-LL catalyst. (b) The influence of Fe content (wt \%) in the ECS-xL catalysts on the productivity and oxygenate selectivity at 2 $\mathrm{hr}$ reaction time of methane to methanol oxidation. Catalytic performance of ECS-LL after consecutive recycling steps with a low (c) and high (d) catalyst loading (Fe mol\%).

XAS analysis suggests that Fe-sites are possibly best described as either isolated Fe centres or multi-Fe segments dispersed evenly through the Al-based octahedral chain, but it cannot unambiguously interpret the second coordination sphere and discriminate between $\mathrm{Al}$ or $\mathrm{Fe}$ neighbours (Al-O-Fe-O-Al, Al-O-Fe-O-Fe or Fe-O-Fe-O-Fe moieties).

The magnetic and structural properties of the catalysts were further assessed by a combined study of electron paramagnetic resonance (EPR) and Mössbauer spectroscopy. Because of the low Fe loading, in order to improve the quality of the Mössbauer analysis, we prepared additional samples using enriched $(95.4 \%){ }^{57} \mathrm{Fe}$. While it proved impossible to fully reproduce the HTS-1 synthesis when starting from ${ }^{57} \mathrm{Fe}$, the electrochemically synthesised samples ${ }^{57}$ ECS-LL (o.5 Fe wt \%) and ${ }^{57}$ ECS-HL (5.4 Fe wt \%) were synthesised and tested in catalysis, demonstrating similar performance to their non-enriched counterparts. From the EPR X/Q-band experiments, it is clear that the HTS samples contain mostly oligomeric Fe species. Indeed, the Mössbauer spectra of the non-enriched ${ }^{56}$ HTS- 1 could be fitted with two components: a dominant magnetic iron $\mathrm{FeO}_{\mathrm{x}}$ cluster and a significantly smaller component, corresponding to a doublet with a QS of 0.98 $\mathrm{mm} \mathrm{s}^{-1}$ and an isomer shift of $0.38 \mathrm{~mm} \mathrm{~s}^{-1}$, typical for high spin $\mathrm{Fe}(\mathrm{III})$ located in a non-centrosymmetric environment (Fig. 3a, Table S4). ${ }^{15}$ In contrast, the degree of dispersion that is observed in the ECS samples is remarkable. An apparent domination of a single atom species can be observed in EPR experiments at 10K for all four ECS samples. The observed X- and Q-band spectra at $10 \mathrm{~K}$ were reasonably fitted with the same set of parameters for an isolated high-spin Fe(III) (Fig. S15). Notably, there is a broad temperature-dependent signal near the central Qband field $(\sim 1200 \mathrm{mT})$, very intense at $\mathrm{T}=150 \mathrm{~K}$ for samples with higher Fe concentration, but also visible for the low Fe content ECS catalysts. The shape and position of this signal (g-factor $\approx 2.0$ ) are similar to those in pure $\mathrm{Fe}(\mathrm{III})$ MIL-53(Fe) (Fig. S16), and zero-field is not detected, suggesting the presence of an extended magnetic $\mathrm{Fe}(\mathrm{III})$ network. A drastic decrease of this signal (virtually reaching zero at $10 \mathrm{~K}$ ) with temperature indicates antiferromagnetic exchange between even number of Fe(III) centres. Remarkably, the estimated $\mathrm{Fe}(\mathrm{III})-\mathrm{Fe}(\mathrm{III})$ exchange 

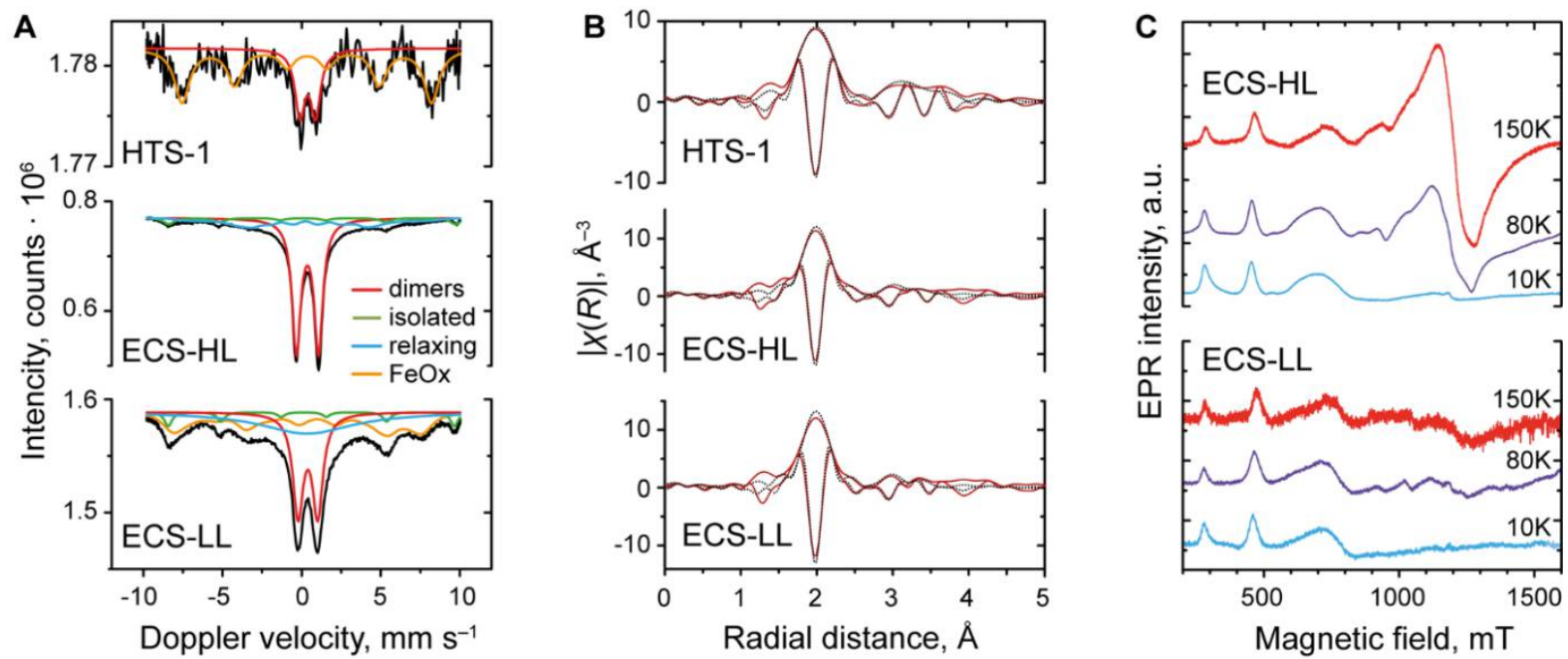

Figure 3. Spectroscopic characterisation of Fe single site. (a) Mössbauer spectra of the HTS-1 and ${ }^{57} \mathrm{ECS}-\mathrm{HL}$ and ${ }^{57} \mathrm{ECS}-\mathrm{LL}$ catalysts in zero field at $4.2 \mathrm{~K}$. The experimental data is represented by back solid lines and the corresponding fitted components are represented by coloured lines. (b) Fourier transform of EXAFS Fe K-edge experimental (red solid line) and fitted (black dots) data for HTS-1, ECS-HL and ECS-LL over the $k$ range 3-11 $\AA^{-1}$ (red, solid line). (c) variable-temperature Q-band $\left(v_{\mathrm{mw}} \approx 33.2-33.8\right.$ $\mathrm{GHz})$ continuous wave (CW) EPR spectra of ${ }^{57} \mathrm{Fe}$ enriched ${ }^{57} \mathrm{ECS}-\mathrm{HL}$ and ${ }^{57} \mathrm{ECS}-\mathrm{LL}$ at $\mathrm{T}=10-150 \mathrm{~K}$. All spectra are normalised to the signal of monomeric $\mathrm{Fe}(\mathrm{III})$ at $\approx 460 \mathrm{mT}$.

coupling $J \sim 100-120 \mathrm{~cm}^{-1}$ (Fig. S17; $\mathrm{H}_{\mathrm{ex}}=J \mathrm{~S}_{1} \mathrm{~S}_{2}$ ) has the same order of magnitude as those previously found for oxobridged $\mathrm{Fe}(\mathrm{III})-\mathrm{Fe}(\mathrm{III})$ dimers in hemerythrin and $\mathrm{E}$. coli ribonucleotide reductase $\left(J=270\right.$ and $220 \mathrm{~cm}^{-1}$, respectively). ${ }^{16}$ Thus, the broad EPR signal with $g \approx 2.00$ is most reasonably assigned to $\mathrm{Fe}(\mathrm{III})$ dimers resembling those in biological systems and additionally coupled by interdimer exchange. In Mössbauer spectroscopy there are no silent Fe species as in EPR and the inter-nuclear magnetic coupling is restricted to lower distances. Thus, the resulting spectra are always composed of all the different species and magnetic hyperfine coupling is mostly observed for interconnected Fe centres. Remarkably, for all the ECS samples, the Mössbauer spectra at $4.2 \mathrm{~K}$ are composed of a dominant doublet component with $\mathrm{QS} \approx 1.4^{-1.2} \mathrm{~mm} \mathrm{~s}^{-1}$ and IS $=0.4 \mathrm{~mm} \mathrm{~s}^{-1}$ (Fig. 3a; Table $\mathrm{S}_{4}$ ) with a small component for single $\mathrm{Fe}(\mathrm{III})$ atoms. From the doublet at $4.2 \mathrm{~K}$ with no magnetic hyperfine structure and, together with the observed EPR temperature dependent signal, it is reasonable to assign the composition of $\mathrm{Fe}$ sites in the ECS samples to a combination of isolated, monomeric $\mathrm{Fe}(\mathrm{III})$ sites and antiferromagnetic coupled dimeric $\mathrm{Fe}(\mathrm{III})-\mathrm{Fe}(\mathrm{III})$ species.

\section{REACTION MECHANISM}

To gain a better understanding of the nature and stability of these catalytic sites, we performed periodic density functional theory (DFT) calculations. Comparison of stability of iron species, incorporated in MIL-53(Al) framework, with extraframework iron clusters show, that species embedded in the MOF structure are significantly more stable (see Table S12). Formation of extraframework Fe species is thermodynamically unfavourable; on the contrary, mixed-metal MIL-53 $(\mathrm{Al}, \mathrm{Fe})$ show considerable stabilization compared to pure MIL-53(Al) and MIL$53(\mathrm{Fe})$ phases. Calculations for monomers, dimers, tri- mers and tetramers of iron in MIL-53(Al) structure show that antiferromagnetically coupled dimeric species are the most stable of them. These results perfectly agree with our EPR and Mössbauer characterization data and further prove the concept of isolated iron active sites, incorporated in the MOF structure.

The reactivity was modelled on isolated Fe species comprising dimeric $\mathrm{Fe}-\mathrm{Fe}$ centres and single monomeric $\mathrm{Fe}$ centres embedded in the MIL-53( $\mathrm{Al})$ structure. For comparison and to understand the origin of the low stability of pure MIL-53 $(\mathrm{Fe})$ (see SI Computational Methods), the respective model was also included in the computational analysis. The analysis of various possible reaction paths allowed us to propose a reaction mechanism schematically illustrated in Fig. 4a. Fig. 4b summarises the results of the DFT calculations and shows the representative structures of the key intermediates involved in the activation of $\mathrm{H}_{2} \mathrm{O}_{2}$ and methane during the catalytic process. For all models, the catalytic cycle starts with the activation of $\mathrm{H}_{2} \mathrm{O}_{2}$ by an Fe site in the MIL-53 structure 1. The most favourable route involves the replacement of one of the carboxylate ligands from the metal node by a hydrogen peroxide molecule. The open carboxylate moiety in $\mathbf{2}$ is stabilised via hydrogen bonding with a bridging hydroxyl group and contains several electrophilic $\mathrm{O}$ sites that, in turn, lead to different pathways for reactivity (see SI Computational Methods). When the number of Fe metal nodes through the framework chain is high and their corresponding carboxylate units are decoordinated, the framework stability is compromised, most likely leading to dissolution of the solid. In samples with sufficiently isolated Fe sites, the subsequent homolytic dissociation of the $\mathrm{O}-\mathrm{O}$ bond $\left(\mathbf{2} \rightarrow \mathrm{H}_{2} \mathrm{O} / 3\right)$ results in the formation of a terminal Fe-OH species that in the case of the MIL-53 $(\mathrm{Fe})$ is very stable $\left(-172 \mathrm{~kJ} \mathrm{~mol}^{-1}\right)$. The concomitant abstraction 


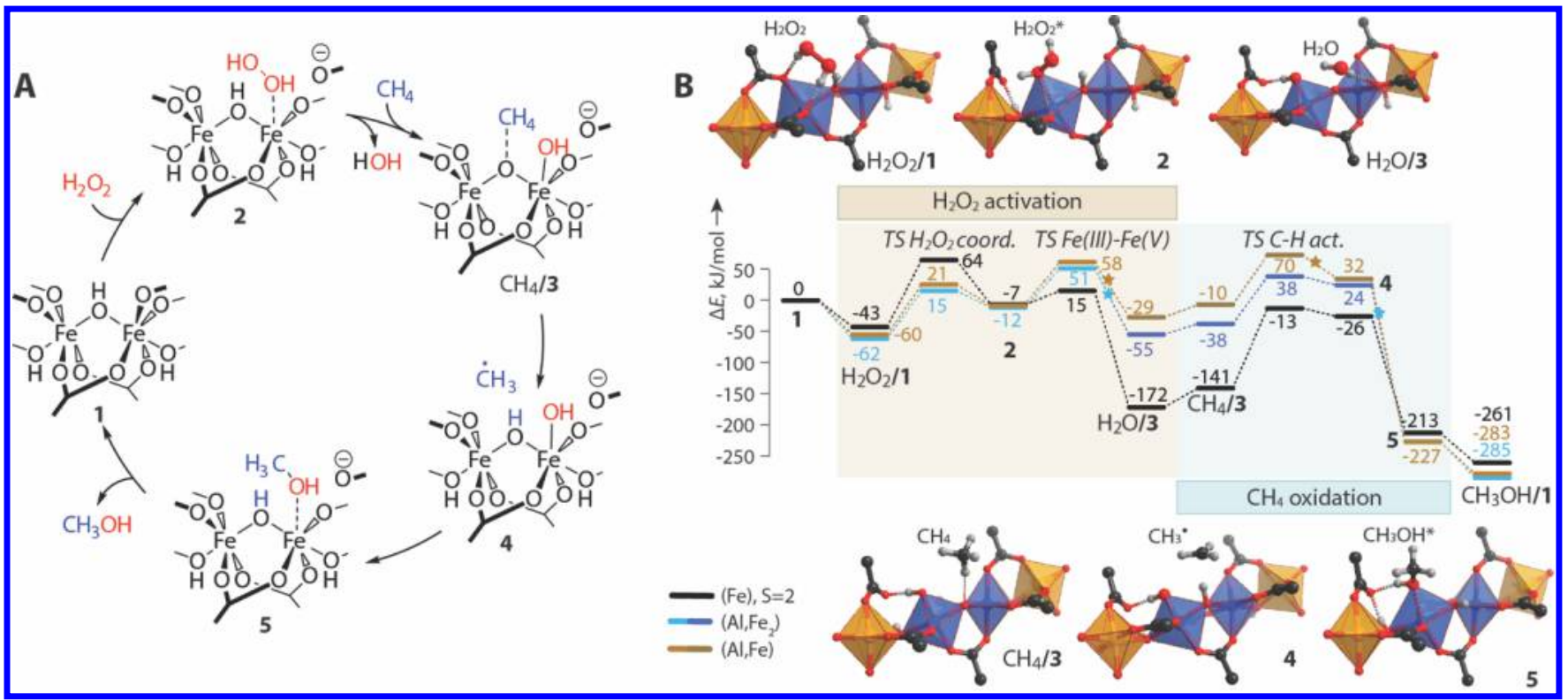

Figure 4. Catalytic reaction mechanism. (a) The proposed mechanism of methane to methanol oxidation with $\mathrm{H}_{2} \mathrm{O}_{2}$ over the dimeric Fe site in MIL-53 $(\mathrm{Al}, \mathrm{Fe})$ and $(\mathbf{b})$ the DFT-computed minimum-energy reaction path diagrams (including spin-transitions indicated with stars) for the catalytic process over different Fe-containing MIL-53 models.

of an $\mathrm{H}$ atom from the bridging hydroxyl group to form $\mathrm{H}_{2} \mathrm{O}\left(\mathrm{H}_{2} \mathrm{O} / 3+\mathrm{CH}_{4} \rightarrow \mathrm{H}_{2} \mathrm{O}+\mathrm{CH}_{4} / 3\right)$ pro-ceeds with a considerable lower energy barriers for isolated Fe sites $\left(\Delta \mathrm{E}^{\ddagger}=80\right.$ and 76 vs $128 \mathrm{~kJ} \mathrm{~mol}^{-1}$ for single Fe(III), Fe(III)$\mathrm{Fe}(\mathrm{III})$ species and MIL-53(Fe) respectively). The formal oxidation state of one Fe centre in the active site increases from (III) to (V). Subsequent homolytic dissociation of the $\mathrm{C}-\mathrm{H}$ bond by the bridging $\mathrm{O}$ site in $\mathrm{Fe}-(\mu-\mathrm{O})-\mathrm{Fe}$ (or $\mathrm{Fe}-(\mu-\mathrm{O})-\mathrm{Al}$ for the single $\mathrm{Fe}$ species) of the activated iron site $\left(\mathrm{CH}_{4} / 3 \rightarrow 4\right)$ yields an $\mathrm{OH}$ group and a $\mathrm{CH}_{3}$ radical which recombines with the terminal $\mathrm{OH}$ ligand to form adsorbed methanol $(\mathbf{4} \rightarrow \mathbf{5})$. Alternatively, reaction with $\mathrm{O}_{2}$ favours the formation of methyl hydroperoxide (Fig. S21). The catalytic cycle is closed and the octahedral ironcarboxylate environment of the active species is restored via the migration of the coordinated methanol to the pore of the MIL-53 $\left(\mathbf{5} \rightarrow \mathrm{CH}_{3} \mathrm{OH} / \mathbf{1}\right)$.

Computational results show that both monomeric and dimeric Fe species in MIL-53(Al) structure can catalyse oxidation of methane with $\mathrm{H}_{2} \mathrm{O}_{2}$. However, lower energy barriers in case of antiferromagnetically coupled dimers as well as their higher stability in the reaction conditions indicate that these species have the highest impact to the overall activity of the catalysts.

\section{CONCLUSIONS}

Based on the extensive spectroscopic characterisation provided here, we conclude that isolated, antiferromagnetically coupled, high spin Fe sites are required to attain a substantial catalytic activity. Control experiments performed using pure phase MIL-53 materials based on redox metals such as $\mathrm{Cr}$ or Fe revealed dissolution of the frameworks under the optimal reaction conditions along with the formation of typical Fenton chemistry products, demonstrating that incorporation of extended $\mathrm{Fe}$ containing moieties within the framework would result in considerable leaching and that the use of a non-redox scaffold is key to obtaining a high catalyst stability, as demonstrated upon recycling of the ECS-LL catalysts (results shown in Fig. $2 \mathrm{c}$ and $2 \mathrm{~d}$ ).

Up to date, many $\mathrm{Fe}_{2} \mathrm{O}$ complexes, trimers and bigger clusters have been characterized. ${ }^{17}$ However, the development of accurate models to describe and mimic the oxo-bridging Fe dimer expressed in enzymes has proven difficult. ${ }^{18}$ Most of these examples can achieve to describe one of the intermediates of the MMO catalytic cycle, especially the highly oxidant intermediate $\mathbf{Q} \cdot{ }^{19}$ However, after $\mathrm{C}-\mathrm{H}$ bond activation, most of them dissociate into monomeric species, ${ }^{20}$ acting as stoichiometric reagents rather than as catalytic sites. As already pointed out by Baerends et al., ${ }^{7}$ a factor to consider when designing biomimetic or bioinspired oxidation catalysts is the ligand field strength. Current models are mostly based on N-rich ligand architectures, which do not fully resemble the carboxylate-bridging diiron sMMO active site and typically yield low spin species rather than the high spin configuration present in the sMMO, with important implications on reactivity. ${ }^{7}$ In contrast to these examples, the MOF based catalysts are, to the best of our knowledge, the first example of isolated Fe dimers that sit on an Obased structure and generate the desired antiferromagnetically coupled high spin species active in the catalytic hydroxylation reaction. Although sMMO enzymes show higher selectivity due to the favourable structure and better site isolation, they demand NADH and additional co-enzymes for regeneration after each catalytic cycle; the use of $\mathrm{H}_{2} \mathrm{O}_{2}$ decreases their activity and leads to protein destruction. On the contrary, MIL-53 $(\mathrm{Al}, \mathrm{Fe})$ catalysts require only $\mathrm{H}_{2} \mathrm{O}_{2}$ for completion of the catalytic process and are stable in the reaction media, representing an important step towards the catalysts for real applications. 
Our findings demonstrate that MOFs are excellent platforms for the development of model catalysts mimicking full enzymatic cycles and open new possibilities for fundamental studies of direct oxidative activation of $\mathrm{CH}_{4}$. We anticipate that the implementation of additional functionalities to allow for the simultaneous activation of oxygen may pave the way to the development of industrially relevant MOF based catalysts.

\section{AUTHOR INFORMATION}

\section{Corresponding Author \\ * Correspondence to: e.a.pidko@tudelft.nl; jorge.gascon@kaust.edu.sa}

\section{Author Contributions}

J.G.S. conceived the idea. J.G.S., A.I.O.S and E.A.P. designed and supervised the research. P.S.C. started the development of the electrochemical synthesis route. A.I.O.S. and D.Y.O. carried out the synthesis, basic characterisation and testing of the different catalysts. A.S. and G.L. carried out DFT calculations. S.L.V. and M.V.F. performed the EPR characterization, I.A.D. performed the Mössbauer characterisation. D.Y.O. analysed the EXAFS data with support from M.A.N. and G.S. A.S. and E.A.P. formulated the mechanistic proposal and analysed the computational data. The paper was primarily written by A.I.O.S. and edited by D.Y.O, E.A.P. and J.G.S. All authors participated in the discussion of the results. †These authors contributed equally.

\section{ASSOCIATED CONTENT}

Supporting Information. Synthesis of MIL-53 $(\mathrm{Al}, \mathrm{Fe})$ catalysts, catalyst testing procedure and product quantification methods, elemental analysis and $\mathrm{CO}_{2}$ physisorption data, TEM and SEM, Raman, XRD, TGA characterization, catalytic results, $1 \mathrm{H}$ NMR spectra of solution after reaction, EXAFS, Mössbauer and EPR characterization, computational results. This material is available free of charge via the Internet at http://pubs.acs.org.

\section{ACKNOWLEDGMENT}

The authors gratefully acknowledge funding from the Dutch National Organization for Scientific Research (NWO), VIDI grant agreement 723.012.107 - MetMOFCat. E.J.M.H. and E.A.P. acknowledge the support from Netherlands Centre for Multiscale Catalytic Energy Conversion (MCEC), an NWO Gravitation programme funded by the Ministry of Education, Culture and Science of the government of the Netherlands. E.A.P. thanks the Government of the Russian Federation (Grant 074-Uo1) for his personal research professorship supported through the ITMO Fellowship and Professorship Program. NWO and SurfSARA are acknowledged for providing access to the supercomputer resources. M.V.F. and S.L.V. thank the Russian Science Foundation (no. 14-13-0o826).

\section{REFERENCES}

1. Nath, I.; Chakraborty, J.; Verpoort, F., Metal organic frameworks mimicking natural enzymes: a structural and functional analogy. Chem. Soc. Rev. 2016, 45, 4127-4170.
2. Labinger, J. A.; Bercaw, J. E., Understanding and exploiting $\mathrm{C}-\mathrm{H}$ bond activation. Nature 2002, 417, 507-514.

3. Zecchina, A.; Rivallan, M.; Berlier, G.; Lamberti, C.; Ricchiardi, G., Structure and nuclearity of active sites in Fe-zeolites: comparison with iron sites in enzymes and homogeneous catalysts. Phvs. Chem. Chem. Phvs. 2007, 9, 3483-99.

4. Sirajuddin, S.; Rosenzweig, A. C., Enzymatic oxidation of methane. Biochemistry 2015, 54, 2283-94.

5. (a) Tinberg, C. E.; Lippard, S. J., Dioxygen activation in soluble methane monooxygenase. Acc. Chem. Res. 2011, 44, 28o-8; (b) Baik, M. H.; Newcomb, M.; Friesner, R. A.; Lippard, S. J., Mechanistic studies on the hydroxylation of methane by methane monooxygenase. Chem. Rev. 2003, 103, 2385-419.

6. Andersson, K. K.; Froland, W. A.; Lee, S. K.; Lipscomb, J. D., Dioxygen independent oxygenation of hydrocarbons by Methane Monooxygenase Hydroxylase Component. New. I. Chem. 1991, 15, 411415 .

7. Kazaryan, A.; Baerends, E. J., Ligand field effects and the high spin-high reactivity correlation in the $\mathrm{H}$ abstraction by non-heme iron(IV)-oxo complexes: A DFT frontier orbital perspective. $\underline{A C S}$ Catal. 2015, 5, 1475-1488.

$8 . \quad$ Sobolev, V. I.; Dubkov, K. A.; Panna, O. V.; Panov, G. I., Selective oxidation of methane to methanol on a FeZSM-5 surface. Catal. Todav 1995, 24, 251-252.

9. (a) Snyder, B. E. R.; Vanelderen, P.; Bols, M. L.; Hallaert, S. D.; Böttger, L. H.; Ungur, L.; Pierloot, K.; Schoonheydt, R. A.; Sels, B. F.; Solomon, E. I., The active site of low-temperature methane hydroxylation in iron-containing zeolites. Nature 2016, 536, 317-321; (b) Narsimhan, K.; Iyoki, K.; Dinh, K.; Roman-Leshkov, Y., Catalytic oxidation of methane into methanol over copper-exchanged zeolites with oxygen at low temperature. ACS Cent. Sci. 2016, 2, 424-9; (c) Grundner, S.; Markovits, M. A. C.; Li, G.; Tromp, M.; Pidko, E. A.; Hensen, E. J. M.; Jentys, A.; SanchezSanchez, M.; Lercher, J. A., Single-site trinuclear copper oxygen clusters in mordenite for selective conversion of methane to methanol. Nat. Commun. 2015, 6 (7546); (d) Hammond, C.; Forde, M. M.; Ab Rahim, M. H.; Thetford, A.; He, Q.; Jenkins, R. L.; Dimitratos, N.; Lopez-Sanchez, J. A.; Dummer, N. F.; Murphy, D. M.; Carley, A. F.; Taylor, S. H.; Willock, D. J.; Stangland, E. E.; Kang, J.; Hagen, H.; Kiely, C. J.; Hutchings, G. J., Direct catalytic conversion of methane to methanol in an aqueous medium by using copper-promoted Fe-ZSM-5. Angew. Chem. Int. Ed. Engl. 2012, 51, 5129-33.

10. Loiseau, T.; Serre, C.; Huguenard, C.; Fink, G.; Taulelle, F.; Henry, M.; Bataille, T.; Ferey, G., A 
rationale for the large breathing of the porous aluminum terephthalate (MIL-53) upon hydration. Chem. Eur. I. 2004, 10, 1373-82.

11. Lee, S. J.; McCormick, M. S.; Lippard, S. J.; Cho, U. S., Control of substrate access to the active site in methane monooxygenase. Nature 2013, 494, 380384 .

12. Martinez Joaristi, A.; Juan-Alcañiz, J.; SerraCrespo, P.; Kapteijn, F.; Gascon, J., Electrochemical synthesis of some archetypical Zn2+, $\mathrm{Cu}_{2+}$, and $\mathrm{Al}_{3}+$ metal organic frameworks. Crvst. Growth Des. 2012, 12, 3489-3498.

13. Beetz, M.; Zimpel, A.; Wuttke, S., Nanoparticles. In The Chemistrv of Metal-Oraanic Frameworks, Wiley-VCH Verlag GmbH \& Co. KGaA: Weinheim, 2016; Vol. I, pp 491-521.

14. Neimark, A. V.; Coudert, F.-X.; Boutin, A.; Fuchs, A. H., Stress-Based Model for the Breathing of Metal-Organic Frameworks. The Iournal of Phvsical Chemistrv Letters 2010, 1, 445-449.

15. Solomon, E. I.; Lever, A. B. P., Inorganic electronic structure and spectroscopy. Wiley: New York, 1999; Vol. I, pp 561-577.

16. (a) Dawson, J. W.; Gray, H. B.; Hoenig, H. E.; Rossman, G. R.; Schredder, J. M.; Wang, R.-H., Magnetic susceptibility study of hemerythrin using an ultrasensitive magnetometer. Biochemistry 1972, 11, 461-465; (b) Petersson, L.; Graslund, A.; Ehrenberg, A.; Sjoberg, B. M.; Reichard, P., The iron center in ribonucleotide reductase from Escherichia coli. J. Biol. Chem. 1980, 255, 6706-12.

17. Friedle, S.; Reisner, E.; Lippard, S. J., Current challenges of modeling diiron enzyme active sites for dioxygen activation by biomimetic synthetic complexes. Chemical Society Reviews 2010, 39, 2768-79. 18. Hohenberger, J.; Ray, K.; Meyer, K., The biology and chemistry of high-valent iron-oxo and iron-nitrido complexes. Nat. Commun. 2012, 3, 720.

19. Kodera, M.; Kawahara, Y.; Hitomi, Y.; Nomura, T.; Ogura, T.; Kobayashi, Y., Reversible O-O bond scission of peroxodiiron(III) to high-spin oxodiiron(IV) in dioxygen activation of a diiron center with a bis-tpa dinucleating ligand as a soluble methane monooxygenase model. I. Am. Chem. Soc. 2012, 134, 13236-9.

20. Xue, G.; Wang, D.; De Hont, R.; Fiedler, A. T.; Shan, X.; Munck, E.; Que, L., Jr., A synthetic precedent for the $\left[\mathrm{FeIV}_{2}(\mathrm{mu}-\mathrm{O})_{2}\right]$ diamond core proposed for methane monooxygenase intermediate Q. Proc. Natl. Acad. Sci. USA 2007, 104, 20713-8.

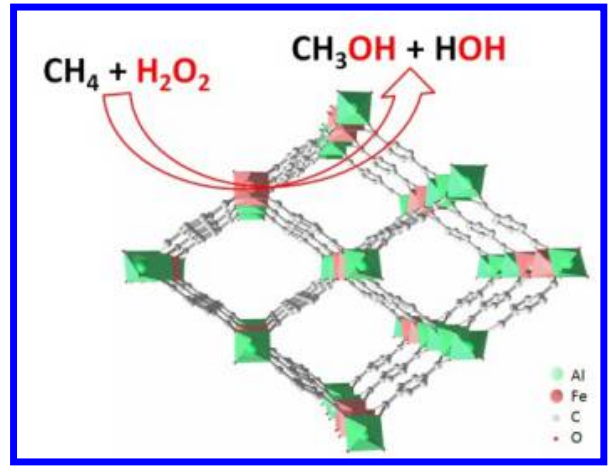

For Table of Contents Only 